\title{
Perlocuciones: ¿reconstrucción o eliminación?
}

\section{(Perlocutions: Reconstruction versus elimination)}

\author{
Antonio Blanco SALgueiro
}

Recibido: 30 de mayo de 2013

Aceptado: 26 de noviembre de 2013

\section{Resumen}

La noción de perlocución ha resultado problemática desde su primera formulación en Austin (1962). Se exploran dos vías diferentes para su clarificación. La vía reconstructiva examina las ambigüedades incrustadas en la caracterización austiniana y trata de deshacerlas mediante taxonomías basadas en criterios precisos. La vía eliminativa, finalmente adoptada, propone sustituirla por una noción alternativa, la de marco situacional de una emisión, capaz de servir mejor al propósito de estudiar "el acto de habla total en la situación de habla total".

Palabras clave: perlocución, ilocución, marco situacional, reconstrucción, eliminación.

\begin{abstract}
The concept of perlocution has been problematic right since its first articulation by Austin. Two possible ways of clarifying the issue are examined. The "reconstructive" way attempts to avoid the ambiguities in the austinian characterization by establishing taxonomies based in precise criteria. The "eliminative" view, finally adopted, seeks the replacement of perlocutions by a concept better fitted for the study of "the total speech act in the total speech situation", the concept of the situational frame of an utterance.
\end{abstract}

Keywords: perlocution, illocution, situational frame, reconstruction, eliminativism. 


\section{Introducción: dos vías alternativas}

Este trabajo quiere esbozar un doble programa para el estudio de la noción de perlocución y de la distinción asociada ilocucionario-perlocucionario, ${ }^{1}$ introducidas ambas como parte del fresh start que Austin (1962) emprende al final de la conferencia VII ante la crisis del contraste inicial, la distinción constatativo-realizativo. No deja de resultar sorprendente, dadas las advertencias de Austin contra las prisas del filósofo por alcanzar soluciones generales antes de explorar pacientemente las sutilidades de los fenómenos de los que quiere dar cuenta, que la noción de perlocución no venga exigida por las reflexiones anteriores, sino que aparezca en la segunda parte del libro "de sopetón” y sin aparente justificación. La dicotomía constatativo-realizativo es reemplazada por la tricotomía locucionario-ilocucionario-perlocucionario, lo que no supone un mero poner tres cosas donde antes había dos. La propuesta inicial era que existen dos clases disjuntas de emisiones, con usos o funciones dispares: representar versus hacer. En la propuesta final, una emisión típica combina ambas funciones, quedando subsumido lo que era el aspecto constatativo dentro del acto locucionario (en el subacto rético) y el aspecto realizativo dentro del acto ilocucionario, a través de la noción de fuerza. ${ }^{2}$ Pero Austin añade que las emisiones sirven para realizar un tercer tipo de actos, actos perlocucionarios. ¿En qué consisten esas cosas adicionales que se pueden hacer con palabras? La caracterización más amplia se da en el momento mismo de su introducción (Austin, 1962, p. 101):

There is a further sense $(C)$ in which to perform a locutionary act, and therein an illocutionary act, may also be to perform an act of another kind. Saying something will often, or even normally, produce certain consequential effects upon the feelings, thoughts, or actions of the audience, or of the speaker, or of other persons: and it may be done with the design, intention or purpose of producing them.

\footnotetext{
1 Empleo los términos "locucionario", "ilocucionario" y "perlocucionario", en lugar de "locutivo", "ilocutivo" y "perlocutivo", que también usan algunos filósofos del lenguaje y lingüistas de habla hispana, para traducir los términos, acuñados por Austin, "locutionary", "illocutionary". y "perlocutionary". Las razones son dos. En primer lugar, se trata de la opción elegida en las traducciones (debidas a filósofos del lenguaje expertos en la materia) de las dos obras fundacionales de la Teoría de los Actos de Habla, How to Do Things with Words (Austin, 1962; traducida por G. Carrió y E. Rabossi) y Speech Acts (Searle, 1969; traducida por L. M. Valdés Villanueva), así como en posteriores traducciones de obras de Searle (Searle, 1983; Searle, 1998). Esas traducciones sientan un importante precedente terminológico, al ser referentes fundamentales para cualquiera que se acerque a la literatura en español sobre los actos de habla. En segundo lugar, no me parece que haya razones idiomáticas profundas para preferir la segunda opción, y, en caso de duda, creo preferible emplear los términos que más se parezcan a los originales, teniendo en cuenta que se trata de términos técnicos.

2 Además, reconoce que podemos constatar de diversos modos (describir, testificar, predecir, afirmar, etc.). Esta variedad se trata ahora como parte de la teoría de la fuerza: estaríamos ante fuerzas diferentes.
} 
Las conferencias VIII, IX y X buscan aclarar el contraste ilocucionario-perlocucionario, que Austin cree que es el que probablemente causará más dificultades (ibid., p. 110). Paradójicamente, la distinción locucionario-ilocucionario fue la más cuestionada con posterioridad. ${ }^{3}$ Tanto las perlocuciones como la distinción ilocución-perlocución suelen aceptarse en la contemporánea Teoría de Actos de Habla (en adelante, TAH), pero resultan problemáticas desde su origen. Pese a sus esfuerzos, Austin no logra establecer un criterio que apuntale la distinción. Existen diversos enfoques acerca de la naturaleza del aspecto ilocucionario de una emisión. ${ }^{4}$ Pero difícilmente se alcanzará una teoría satisfactoria de las ilocuciones sin una visión clara de sus fronteras. Las perlocuciones han recibido relativamente poca atención dentro de la TAH. ${ }^{5}$ Existe una tendencia a tratar lo perlocucionario como "cubo de basura" de lo ilocucionario, según el esquema: "Si usted tiene una teoría de las ilocuciones y se enfrenta a un caso problemático, trátelo como una perlocución". ${ }^{6} \mathrm{Sin}$ embargo, la comprensión del fenómeno es urgente si nos tomamos en serio el lema austiniano según el cual "el acto de habla total en la situación de habla total es el único fenómeno real que, en última instancia, estamos tratando de elucidar." (ibid., p. 148). Sea lo que sea exactamente aquello a lo que apunta la noción, no puede ser soslayado por quien adopte una aproximación al lenguaje que asuma la tarea de estudiarlo en toda la complejidad de sus funciones en la vida humana.

A continuación se exploran dos vías posibles, aparentemente incompatibles, para tratar con esta situación, a las que denomino "reconstructiva" y "eliminativa". Aunque finalmente se apostará por la segunda vía, el examen de la primera permite localizar los problemas y justificar la necesidad de una alternativa. La opción reconstructiva parte de la impresión de que la noción austiniana es vaga porque abarca aspectos heterogéneos del "acto de habla total". El reconstructivista asume la tarea de distinguir todos los aspectos involucrados, reconstruyendo una o varias

\footnotetext{
3 Véanse, por ejemplo, Cohen (1969), Searle (1973) y Strawson (1973). Cohen considera que la fuerza no es algo distinto del significado literal. Searle elimina la noción de acto locucionario, sustituyéndola por la de acto proposicional (que incluye actos de referencia y predicación), y retiene las nociones de acto ilocucionario (que incluye al anterior) y acto perlocucionario.

4 Blanco Salgueiro (2004) examina críticamente diversas teorías acerca de la fuerza ilocucionaria.

5 Las perlocuciones recibieron cierta atención en los años 70 del s. XX (Cohen, 1973; Campbell, 1973; Gaines, 1979; Davis, 1979). Más recientemente, Gu (1993) adopta una postura eliminativista. Cavell (2005) retoma el tema para discutir las "emisiones apasionadas". Otras obras discuten el carácter ilocucionario o perlocucionario de actos como amenazar (Nicoloff, 1989) o incitar (Kurzon, 1998). En la filosofía feminista se ha debatido si la subordinación de la mujer que produce la pornografía es un efecto perlocucionario o es constitutiva de una ilocución (Hornsby, 1993; Langton, 1993; Hornsby \& Langton, 1998). Blanco Salgueiro (2008) sugiere pruebas para distinguir (entre las emisiones "hostiles") las ilocuciones de las perlocuciones.

${ }^{6}$ La estrategia se inaugura en Srawson (1964), quien considera que jactarse es una perlocución porque no encaja en la teoría intencionalista griceana que considera la base explicativa de la fuerza de una emisión.
} 
nociones útiles para fines teóricos específicos, y trata de aclarar sus relaciones mutuas. El resultado final podría incluir una taxonomía de las perlocuciones elaborada de acuerdo con principios sistemáticos.

Una opción menos conservadora parte de la sospecha de que la noción de perlocución surge de una comprensión deficiente de la estructura del "acto de habla total en la situación de habla total" (en adelante, AHT-SHT). Esta vía es escéptica con respecto a la utilidad teórica de la noción, pero no tiene por qué limitarse a proponer su eliminación (esa podría ser sólo la pars destruens de la propuesta). Nuestra hipótesis será que los fenómenos a los que apunta la noción austiniana son importantes y deben ser abarcados por una teoría suficientemente holística del uso del lenguaje. La idea es que la noción debe ser, en último término, sustituida por alguna otra mejor perfilada y fundamentada. La verdadera eliminación se produce, en la TAH, por la simple falta de interés en las perlocuciones, que son sistemáticamente relegadas o ignoradas en la práctica. Aquí no se tratará de negar ciertos fenómenos relacionados con el uso del lenguaje, sino de modificar el entramado teórico que utilizamos para dar cuenta de ellos.

El desinterés por las perlocuciones se origina ya en Austin, que las considera sólo para distinguirlas de las ilocuciones, que son el foco principal de su atención. De hecho, no intenta clasificarlas, al contrario de lo que hace con las locuciones y las ilocuciones (Cerf, 1969, p. 354). Los autores posteriores repiten los escasos ejemplos de Austin, contribuyendo a la impresión de que no se trata de un territorio rico y variado ${ }^{7}$.

Los estudiosos en este ámbito han solido adoptar una de las dos vías esbozadas: o bien tratan de perfilar la noción, eliminando sus ambigüedades, con el objetivo de construir una teoría de las perlocuciones, a menudo acompañada de una taxonomía de las mismas ( $c f r$. Cohen, 1973; Gaines, 1979; o Davis, 1979), o bien adoptan un punto de vista más bien escéptico, como sucede en Campbell (1973), Gu (1993) o Kurzon (1998).

En el apartado 2 se despliegan las principales ambigüedades que aquejan a la noción austiniana y algunas soluciones que podría aportar un "reconstruccionista"

\footnotetext{
${ }^{7}$ Cabe contrastar (una posible línea taxonómica entre otras muchas) los usos "benéficos" del lenguaje, en los que se mejora el estado de la audiencia, con los "negativos", en los que se empeora su situación. Los primeros incluyen alegrar, alertar, animar, calmar (apaciguar, tranquilizar), consolar, emocionar, distraer, divertir, embelesar, encantar, fascinar, hacer feliz, instruir, etc. Los segundos incluyen aburrir (producir tedio), acobardar, alarmar, asustar, atemorizar, avergonzar, confundir, decepcionar, deprimir, desanimar, desconcertar, desconsolar, descorazonar, disgustar, enfadar (soliviantar), exasperar, enervar, entristecer, frustrar, inquietar, intimidar, irritar, ofender, ofuscar, etc. Otras muchas "perlocuciones" son neutrales al respecto, como convencer, exaltar, disuadir, intrigar, persuadir, sorprender, etc. No estamos ante una lista de verbos que sean esencialmente de actos de habla, sino que tienen un uso perlocucionario: a veces hacemos esas cosas con palabras $\mathrm{y}$, en esa medida, pueden interesar a la TAH.
} 
para desambiguarla con el fin de obtener una caracterización mejor fundada. A continuación (apartado 3.1), se argumenta que la noción de perlocución genera una tensión entre dos principios austinianos básicos, el principio de realizatividad generalizada (RG) y el principio holista (H). Se explora entonces (apartado 3.2) la posibilidad de afrontar los problemas sustituyendo la noción de perlocución por la de marco situacional de una emisión, y se formulan tres argumentos para preferir la nueva noción como concepto teórico básico de la TAH (apartado 3.3). Finalmente, se recapitula la argumentación y se sugiere la posibilidad de conservar un espacio teórico limitado para las perlocuciones.

\section{Reconstrucción}

Tras introducir las perlocuciones, Austin dedica sus energías a buscar criterios que apuntalen la distinción ilocucionario-perlocucionario. Parte del esfuerzo asume que la distinción está ya marcada en el lenguaje corriente, al que su metodología otorga la "primera palabra" (Austin, 1956/57). Un primer intento apela a la distinción entre las preposiciones usadas comúnmente en inglés para referirse a los actos de (of) decir algo (locucionarios), frente a los realizados al (in) decir algo (ilocucionarios) y a los que se realizan por (by) decir algo (perlocucionarios). Pero enseguida rechaza este criterio por insuficiente, ya que la gente no es consistente al usar esas preposiciones para describir lo hecho con palabras, y además las emplea para señalar cosas que quedan fuera de la taxonomía, como cuando se dice que al decir algo alguien estaba bromeando. 8

Una segunda forma de apelar al lenguaje corriente parte de que la distinción está sedimentada en él en dos tipos de verbos que funcionan de modos diferentes. Los primeros pueden ser usados en "forma realizativa" (primera persona del presente simple de indicativo, voz activa) para explicitar los actos en cuestión. Puedo prometer diciendo "Prometo...", o advertir diciendo "Te advierto...". Los segundos no se usan de ese modo, pues no se convence a alguien por decir "Te convenzo...", ni se le ofende por decir "Te ofendo". Austin liga este criterio a lo que considera la marca fundamental de lo ilocucionario: su dependencia del rol convencional de las palabras. Su interés central es estudiar cómo hacemos cosas con palabras en virtud de convenciones asociadas con ellas, lo que puede explicar su relativo desinterés por las perlocuciones. Sin embargo, las nociones de convención y de actuar de acuerdo con una convención no son aclaradas, y muchos griceanos han defendido posteriormente que las intenciones son más importantes que las convenciones a la

\footnotetext{
8 Con anterioridad, Austin mismo ha empleado in para caracterizar la relación entre el acto fonético y el fático: "in performing one I am performing the other" (Ibíd., p. 96).
} 
hora de explicar el aspecto ilocucionario de una emisión. ${ }^{9}$ Esta marca de la distinción en el lenguaje corriente está relacionada con la teoría de la acción presupuesta cuando decimos que al hablar realizamos varios actos entrelazados que mantienen complejas relaciones de dependencia mutua. Cuando se trata de acciones no verbales, comúnmente es arbitrario y dependiente de nuestros intereses separar lo que alguien hace de sus consecuencias. Así, puedo decir que alguien movió un dedo y como consecuencia el gatillo se movió, o que apretó el gatillo y como consecuencia el arma se disparó, o que disparó el arma y como consecuencia murió el burro, etc. (Austin, 1962, pp. 107-108). Pero en el caso de las acciones verbales, disponemos de la ayuda de la "nomenclatura" que nos proporciona la existencia de esas dos clases de verbos que parecen diseñadas para marcar la distinción "en un punto regular" entre el acto verbal básico realizado y otras cosas que se conectan más lejanamente con la emisión. Ambas clases de verbos se coordinan en comentarios en los que una acción verbal se supedita a otra, como "Informó a $o$ de que $p$ y así le convenció de que q"; "Le prometió que $p$ y por ello lo alegró"; "Insultó a o con lo cual lo hirió profundamente, etc.

Los varios tipos de ambigüedad que los críticos post-austinianos han detectado en los comentarios de Austin sobre las perlocuciones pueden ser clasificados en tres apartados: las que conciernen a los efectos constitutivos de un acto perlocucionario; las que se refieren a las causas de las perlocuciones; y las que afectan a la naturaleza de los mecanismos que se supone que conectan o vinculan las causas con los efectos.

\subsection{Efectos perlocucionarios}

En cuanto a los efectos constitutivos de una perlocución cabe discutir, en primer lugar, si se reducen a efectos sobre el oyente o deben incluirse efectos sobre el hablante $\mathrm{u}$ otras personas, e incluso efectos ulteriores sobre el mundo físico y social. En el citado pasaje inicial, Austin admite las tres primeras posibilidades (y no excluye otras), pero sus ejemplos son todos de efectos sobre la audiencia, y los autores posteriores también suelen soslayar otras clases de efectos ( $c f r$. Gaines, 1979, p.

\footnotetext{
9 Bach y Harnish (1979) es un caso paradigmático. Los griceanos suelen apelar a la noción de convención de D. Lewis (Lewis, 1969) para explicar los casos en los que el significado (y la fuerza) se vehiculiza a través de convenciones lingüísticas, pero no consideran que tales convenciones sean, en general, necesarias para que una emisión posea una fuerza paradigmática. Davis (1979) propone sustituir la vaga noción de convención por la noción chomskyana de competencia lingüística, ampliándola para incluir la competencia pragmática en las reglas para el uso de la lengua. Pero en muchos ejemplos austinianos la noción de convención presupuesta hace referencia a la competencia social (y no sólo lingüística) de los protagonistas. Así, para casarse por la Iglesia diciendo "Sí quiero" hay que participar en una forma de vida, y no sólo conocer las reglas de uso del castellano.
} 
207). Pero el habla produce efectos sobre los hablantes que a menudo son reformulables en el lenguaje de la acción:10 "Por (/al) insultarlo, me desahogué", "Por confesar el crimen, me alivié". Y el habla puede tener efectos terapéuticos ("es bueno hablar las cosas"). También es evidente que a través del habla se producen efectos ulteriores sobre terceras personas y sobre el mundo y que, si no se quieren tratar como parte del "acto de habla total" (en particular, como efectos perlocucionarios) debe explicarse por qué. Al decir que Bush atacó Irak le atribuimos una acción que es desencadenada por una ilocución suya, su orden de atacar.

En cuanto al tipo de efectos involucrados, Austin distingue entre sentimientos, pensamientos y acciones, pero la enumeración no parece pretender ser exhaustiva y seguramente admitiría otros tipos de estados mentales (de la audiencia, del hablante o de terceras personas). Ello puede conducir a una taxonomía de las perlocuciones basada en el tipo de efecto mental producido ( $c f r$. Gaines, 1979).

Las cosas se complican cuando al final de la conf. IX Austin afirma que algunos efectos que conforman el acto de habla total forman parte del aspecto ilocucionario, y explicita tres de esos tipos de efectos ilocucionarios: la comprensión (uptake) por parte del oyente; que la emisión "tenga efecto"; y la invitación a una respuesta. En primer lugar, el éxito ilocucionario no queda nunca unilateralmente garantizado por el hablante, sino que el oyente debe contribuir al menos con su comprensión del significado y de la fuerza de la emisión; si $h$ dice "Vendré mañana" y $o$ no lo toma como una promesa, $h$ no ha prometido. Segundo, las ilocuciones tienen un efecto convencional, como cuando un bautismo acarrea que ciertas prácticas de denominación sean legítimas y otras no, o como cuando nombrar a alguien para un cargo produce que esa persona lo ocupe desde entonces y que (si es unipersonal) ninguna otra pueda ocuparlo mientras ella lo ocupe. Por último, según Austin muchas ilocuciones se vinculan esencialmente a la producción de efectos en el sentido de invitar a una respuesta o secuela por parte del oyente o de hacer esperable una acción ulterior del hablante. La idea es que no es posible caracterizarlas de modo exhaustivo sin mencionar ciertos efectos con los que están íntimamente liga-

\footnotetext{
10 A veces no son reformulables de ese modo, o lo son sólo a costa de forzar mucho las cosas; por ejemplo, al decir "Cuando hablo de mis padres me emociono", emocionarme no parece aquí algo que hago, sino más bien algo que sufro. La propuesta que se esbozará en el apartado 3 permite incluir dentro de un enfoque teórico alternativo los efectos del habla que no parecen reducibles a acciones del hablante. Austin rechaza considerar como parte de lo perlocucionario los efectos resultantes de canalizar emociones verbalmente, seguramente porque éstos no son intuitivamente parte de lo que el hablante hace (Austin, 1962, p. 105). Este punto centra la crítica de Cavell (2005), que propone un estudio sistemático de las "passionate utterances" a través de una reconstrucción de la noción de perlocución. Cabe preguntar qué efectos sobre el hablante prevé Austin en el pasaje en el que introduce la noción de perlocución, si quiere excluir los efectos más obvios que una emisión puede desencadenar en la mente del emisor. Tal vez pensase en casos en que uno se convence a sí mismo dándose argumentos, o se anima a sí mismo, etc.
} 
das. Por ejemplo, ¿cómo explicar qué es protestar sin aludir a que las protestas buscan una rectificación en las acciones o actitudes de aquellos a los que van dirigidas, o qué es ordenar sin mencionar los actos de obediencia perseguidos? ${ }^{11}$ Este punto es el que más amenaza la pretensión de trazar nítidamente la frontera ilocucionarioperlocucionario. ${ }^{12}$ Cohen (1973) parece cuestionar que los efectos de esa tercera clase sean ilocucionarios, al distinguir tres tipos de efectos perlocucionarios: a) Los producidos por una locución, sin la intervención de aspectos propiamente ilocucionarios, como cuando $h$ dice " ¡No te despiertes!" en un tono que causa que $o$ se despierte (causa fonética); o cuando $h$ llama la atención de $o$ al pronunciar su nombre mientras habla con un tercero (causa rética). b) Los producidos por una ilocución (causa ilocucionaria), pero no están asociados a ella, como sorprender, asustar, exasperar, divertir, aburrir o fascinar en relación a afirmar. No es preciso referirse a esos efectos para caracterizar la correspondiente ilocución: al afirmar algo puedo asustar o divertir a mis oyentes, pero esos efectos no tienen nada que ver con lo que en esencia consiste afirmar algo. c) Por último, hay efectos asociados con una ilocución: intimidación con amenazar, alertar con advertir, persuadir/disuadir con argumentar, obedecer (lograr que o obedezca) con ordenar, o responder (lograr que o responda) con preguntar. ${ }^{13}$ Esos efectos parecen incluir los involucrados en el tercer sentido en el que según Austin las ilocuciones están vinculadas constitutivamente a la producción de efectos. El propósito de producirlos debe ser mencionado para caracterizar de modo completo la correspondiente ilocución, pues constituye un objetivo (point) de la misma. De hecho, la teoría de los infortunios se aplica aquí. Hay algo muy desafortunado en una pretendida protesta que no quiera cambiar nada, en una orden que no pretenda ser obedecida, o en una amenaza que no pretenda intimidar. ${ }^{14}$ Es cierto que lo constitutivo parece ser más el propósito (con-

11 No se quiere decir que los efectos "constitutivos" en cuestión deban estar presentes en cada caso particular (una orden desobedecida es una orden), sino algo similar a lo que dice Wittgenstein en el § 345 de las Investigaciones filosóficas, donde se afirma que aunque las órdenes no son obedecidas a veces, si nunca fuesen obedecidas el concepto 'orden' perdería su propósito. En realidad, se puede decir algo más fuerte: que aunque una orden nunca fuese obedecida, al menos el propósito de ser obedecida no es desligable del concepto de dar una orden.

12 Un problema adicional, que se hará más evidente cuando introduzcamos la noción de marco situacional, es que obedecer o responder (frente a intimidar o alertar) no se dejan tratar bien como actos (a fortiori, como actos perlocucionarios) del emisor, sino que piden a gritos ser tratados como actos de la audiencia.

13 Cohen (1973, p. 497) establece la noción de "perlocución asociada" del siguiente modo, bastante vago: "An associated perlocution is one brought about by means of an illocution it 'belongs with'. Examples are intimidating, which is associated with threatening [...] alerting, which is associated with warning, and convincing and persuading, which are associated with arguing".

14 Esto se adelanta en la conf. 2. La condición $\Gamma 2$ sobre los realizativos indica que los participantes en el procedimiento convencional "Must actually so conduct themselves subsequently". Quien promete, apuesta o contrata, debe conducirse de modo consecuente, quien acata una orden debe obedecer, etc. 
vencional) de producir el efecto que su producción efectiva. Puedo amenazarte exitosamente aunque, de hecho, no te intimide, $u$ ordenarte que hagas algo sin que obedezcas. Pero lo importante es que no es posible separar el estudio de ciertas ilocuciones del de ciertas "perlocuciones": para analizar por completo las primeras debo mencionar las segundas. Ciertamente, $h$ puede intimidar a $o$ tanto con una amenaza como con un informe, o puede convencer a $o$ de que $p$ tanto con un informe (o un argumento) como con una amenaza. Pero las amenazas están asociadas a la intimidación de un modo más íntimo y diferente a como a veces se conectan con la producción de convencimientos. Las amenazas son inherentemente hostiles, mientras que los informes sólo resultan hostiles contingentemente y en contextos especiales. Por su parte, informar o argüir están asociados con convencer al oyente de un modo más íntimo y diferente a como a veces se conectan con intimidarlo.

Para reconstruir todas las nociones involucradas hasta ahora, deben establecerse varias taxonomías paralelas. Según los destinatarios de los efectos, habrá perlocuciones dirigidas al oyente $\left(\mathrm{PER}_{o}\right)$, al hablante $\left(\mathrm{PER}_{h}\right)$, a terceros $\left(\mathrm{PER}_{t p}\right)$, y al mundo externo, físico y social $\left(\mathrm{PER}_{m}\right)$. Según el tipo de efecto podemos distinguir las que resultan en acciones $\left(\mathrm{PER}_{a c}\right)$, en sucesos mundanos $\left(\mathrm{PER}_{\text {suc }}\right)$, o en estados mentales $\left(\mathrm{PER}_{m e n}\right)$. Este último grupo se puede subdividir de acuerdo con una taxonomía de tipos de estados mentales (deseos, creencias, intenciones, diversas clases de sentimientos y emociones, etc.). Por último, según la estrechez del vínculo del efecto con la causa, cabe distinguir las perlocuciones asociadas a la causa $\left(\mathrm{PER}_{a s o c}\right)$ de las no asociadas $\left(\mathrm{PER}_{\text {no-asoc }}\right){ }^{15}$

\subsection{Causas perlocucionarias}

En cuanto a las causas perlocucionarias, podemos preguntarnos si siempre son ilocuciones, o pueden contar también las locuciones. Cohen (1973) llama (extrañamente) oblicuas a las perlocuciones producidas a partir del acto locucionario en cualquiera de sus aspectos (fonético, fático o rético), y directas a las producidas a partir del acto ilocucionario (con independencia de que estén asociadas a la ilocución). Podemos establecer entonces una nueva clasificación de las perlocuciones, que distinga entre las locucionariamente inducidas $\left(\mathrm{PER}_{l o c}\right.$, en sus tres modalidades, fonética: $\mathrm{PER}_{\text {fon }}$, fática: $\mathrm{PER}_{\text {fat }} \mathrm{y}$ rética: $\left.\mathrm{PER}_{\text {ret }}\right)^{16} \mathrm{y}$ las inducidas ilocuciona-

15 Para la última clasificación hay que considerar tanto el efecto como la causa, ya que, como hemos visto, intimidar o convencer sólo son perlocuciones asociadas o no asociadas en relación a ciertas ilocuciones.

16 Si se elige el marco searleano (que prescinde de las locuciones) hay que distinguir entre perlocuciones inducidas por el acto emisivo $\left(\mathrm{PER}_{e m}\right)$ e inducidas por el acto proposicional ( $\left.\mathrm{PER}_{\text {prop}}\right)$, distin- 
riamente $\left(\mathrm{PER}_{i l o c}\right)$. Además, una perlocución puede producir efectos ulteriores, por lo que habría que admitir perlocuciones inducidas perlocucionariamente $\left(\mathrm{PER}_{p e r}\right)$. Así, al afirmar que $p$ puedo convencerte de que $q\left(\mathrm{PER}_{i l o c}\right)$, y por ello conseguir enfadarte $\left(\mathrm{PER}_{\text {per }}\right){ }^{17}$

El que las causas perlocucionarias deban considerarse como una parte integral de toda genuina perlocución se sigue de la idea austiniana de que ciertos efectos que a veces son perlocucionarios se consiguen en muchas otras ocasiones a través de una variedad de medios no verbales. Puedo divertir a alguien diciendo algo gracioso, pero también haciendo piruetas; no obstante, divertir sólo cuenta teóricamente como una perlocución cuando se consigue verbalmente (o, al menos, comunicativamente).

\subsection{El vínculo perlocucionario}

¿Cómo logra una causa perlocucionaria producir un efecto perlocucionario? Un primer aspecto de esa cuestión se discute en $\mathrm{Gu}$ (1993), quien señala una ambigüedad con relación a la naturaleza del vínculo causal, entre tres sentidos: 1) causación fisico-mecánica, como cuando digo "¡No te despiertes!" en un tono tal que te despierto; 2) causación verbal, que opera a través de la comprensión del lenguaje; 3) causación verbal-influencial, en la cual el efecto causado no se asigna sólo al acto ilocucionario realizado, sino también a factores como las relaciones interpersonales, las relaciones de poder (políticas, administrativas o académicas), los motivos, y otras contingencias, como cuando $o$ reacciona a la emisión "Es inminente un terremoto" convenciéndose de que se avecina un terremoto, basándose no sólo en la comprensión de lo que $h$ dice, sino en que cree que $h$ es un experto sismólogo. La cuestión del papel de las convenciones frente a las razones en la producción de los efectos perlocucionarios es tratada marginalmente por Austin, al final de una nota a pie de página (Austin, 1962, p. 113):

the sense in which saying something produces effects on other persons, or causes things, is a fundamentally different sense of cause from that used in physical causation by pressure, \&c. It has to operate through the conventions of language and is a matter of influence exerted by one person on another: this is probably the original sense of 'cause'.

guiendo entre las últimas las inducidas por actos de referencia $\left(\mathrm{PER}_{r e f}\right)$ y por actos de predicación $\left(\mathrm{PER}_{\text {pred }}\right)$.

17 Este sería un modo de tratar la distinción austiniana entre perlocuciones de tipo C.a y C.b., en secuencias como: "Me ordenó dispararle" (ilocución), "Me persuadió a dispararle" (perlocución C.a.) y "Consiguió que le disparase" (perlocución C.b.). (Austin, 1962, p. 102). 
Austin se inclina ahí por el tercer sentido de "causa" entre los señalados por Gu, y excluye el primero. Eso deja fuera algunas perlocuciones locucionariamente inducidas, pero no permite distinguir, dentro de las "directas", las asociadas de las no asociadas, ya que al advertir a $o$ de que $p$, invocando tanto su influencia como las convenciones del lenguaje, $h$ puede tanto alertar a $o$ (posible $\mathrm{PER}_{\text {asoc }}$ ) como sorprenderlo $\left(\mathrm{PER}_{\text {no-asoc }}\right) .18$

En principio, si no hay presiones teóricas que lo desaconsejen, un partidario de la reconstrucción puede tratar de dar cuenta de todos los fenómenos que están en juego, mediante una nueva taxonomía de acuerdo con el mecanismo causal que interviene en las perlocuciones, distinguiendo las causadas físico-mecánicamente $\left(\mathrm{PER}_{f i s}\right)$ de las que se producen a través de la comprensión del lenguaje $\left(\mathrm{PER}_{\text {comp }}\right)$ y de las que se producen a través de la consideración de razones o de la influencia ejercida por el emisor $\left(\mathrm{PER}_{\text {inf }}\right)$.

Un segundo aspecto concerniente al vínculo perlocucionario es si la causación debe ser intencional o puede no serlo, como admite Austin en el pasaje inicial, donde dice que el acto perlocucionario puede realizarse con el designio, intención o propósito de producir los efectos en cuestión. Gaines (1979) piensa que tomar como constitutivo de una perlocución el respaldo de una intención de producir el efecto es lo que permite atribuir esos actos al emisor. Pero esa tesis presupone una concepción nada austiniana acerca de la responsabilidad sobre las acciones, pues Austin sostiene en diversos lugares que debemos distinguir siempre entre intentar hacer algo y conseguirlo, y que a menudo hacemos cosas sin pretenderlo. Gaines quiere que la TAH admita como perlocuciones sólo acciones producidas intencionadamente a partir de ilocuciones. Con ello se lograría cierto control teórico, pero a costa de eliminar elementos importantes del AHT-SHT. Así, si digo: "Le prometí

18 El caso en el que $h$ despierta a $o$ gritando: “iDespierta!” es distinto de aquel en el que atrae su atención al mencionar su nombre, donde el efecto se logra por medio de la comprensión. Por eso, Davis (1979) no considera perlocucionarios los efectos del primer tipo pero sí los inducidos por aspectos proposicionales. Sin embargo, los efectos de los distintos aspectos locucionarios son un fenómeno interesante dentro del AHT-SHT. En el siguiente pasaje, es el tono de una voz el que impresiona al personaje, pero la causación no es puramente mecánica, sino que se basa en un razonamiento del oyente. Esa posibilidad cuestiona el presupuesto, común a T. Cohen y Gu, de que si la causa es fonética la causación debe ser físico-mecánica:

"Esperé unos segundos, y cuando estuve seguro de que había subido a la planta alta, me acerqué de puntillas hasta el pie de la escalera y agucé el oído. / Oí la voz de Kennedy. En voz baja explicaba el proyecto. Se entendían palabras sueltas. Luego, un momento de silencio, y la voz de una mujer.

-Muy bien, si usted cree que no hay riesgos.

Pero no me impresionó el sentido de las palabras, sino la voz misma. Yo conocía aquella voz, con sus matices duros, fríos, metálicos. / La amiga del coronel Kennedy era la mujer que me había llamado dos veces. La mujer que me enviara cinco mil dólares." (J. Hadley Chase: Una corona para tu entierro) 
casarme con ella tratando alegrarla pero sólo logré deprimirla", habría que considerar lo primero como un acto perlocucionario fallido y lo segundo como algo sin interés para la TAH. Pero eso es arbitrario. El hecho de que la deprimí es interesante porque es un principio de prudencia pragmática que debo cuidar lo que digo, medir mis palabras. Además, en una conversación las metas perlocucionarias no se logran siempre a la primera y continuamente se obtienen efectos imprevistos que, si son adversos, pueden obligarnos a pedir excusas. En una línea afín a la de Gaines, Bach \& Harnish (1979) afirman que la pragmática sólo se ocupa de los objetivos comunicativos del emisor, por lo que abogan por un intencionalismo perlocucionario. Los autores defienden a su vez un intencionalismo ilocucionario de corte griceano, según el cual toda ilocución está repaldada por una intención compleja. En cambio, Austin rechazó fundamentar la teoría de la fuerza en la mente del emisor (la suya es una teoría convencionalista de la fuerza). Pero si se renuncia al intencionalismo ilocucionario no hay razón para aceptar el perlocucionario, pues las ilocuciones están claramente bajo un mayor control intencional que las perlocuciones. 19 Puedo asustarte sin querer, y aquí es fácil dar y aceptar excusas, pero para que admitas que te ordené algo sin querer me hará falta una buena historia. Davis (1979) caracteriza las perlocuciones de modo que quepan tanto las respaldadas por intenciones como las no intencionadas, lo que sugiere una nueva taxonomía según estén causadas intencionadamente $\left(\mathrm{PER}_{\text {int }}\right)$ o no $\left(\mathrm{PER}_{\text {no-int }}\right) \cdot{ }^{20}$

Las ambigüedades señaladas en los apartados $2.1,2.2$ y 2.3 dan una idea de cuán heterogéneo es el concepto austiniano de perlocución y, por ello, de la compleja tarea a la que se enfrenta quien opte por la vía reconstructiva. Sin embargo, la tarea podría verse facilitada o restringida si se explicita el papel explicativo que se supone que deben desempeñar las perlocuciones, o se aclaran las razones por las cuales se las invoca. La reivindicación de algunos de los fenómenos más específicos enredados en la madeja de lo perlocucionario podría venir de perspectivas o marcos teóricos concretos que justifiquen la necesidad de prestarles atención diferenciada. De ese modo, algunas de las nociones que hemos distinguido podrían ser juzgadas como no pertinentes para ningún propósito teórico serio, lo que disminuiría la complejidad de la tarea reconstructiva. 21

19 Austin admite que se puede realizar de modo no intencionado un acto ilocucionario: "I may order someone to do something, when I did not intend to order him to do so", pero añade enseguida que esa posibilidad es más clara en el caso de las perlocuciones: "But it is in connection with perlocution that it is most prominent, as is also the distinction between attempt and achievement." (Austin, 1962, p. 106).

20 Podría ser necesaria una ulterior subdivisión dentro de $\mathrm{PER}_{\text {int }}$, ya que $h$ puede querer realizar o bien una perlocución subrepticia (i. e., tratando de evitar que $o$ reconozca la intencionalidad de la perlocución), o bien una perlocución abierta (i. e., tratando de que su intencionalidad sea reconocida).

21 Campbell (1973) establece una conexión entre el estudio de las perlocuciones y la retórica ( $c f r$. también Gaines, 1979, p. 208). La noción se puede aplicar al estudio de las terapias verbales. Existen posi- 


\section{Eliminativismo perlocucionario}

Aunque es posible que la noción austiniana puede ser mejorada y reivindicada a través de la reconstrucción, a continuación se argumentará que es preferible una salida más radical: la eliminación de las perlocuciones y su sustitución por una noción teórica alternativa más adecuada para el propósito de elucidar el AHT-SHT.

\subsection{Realizatividad generalizada vs. holismo austiniano}

El abandono de la distinción inicial (constatativo-realizativo) y el recomienzo emprendido por Austin al final de la conf. VII suponen la defensa de lo que puede denominarse una realizatividad generalizada. Si al principio sostenía que a veces hablar es actuar, finalmente va a asumir que hablar siempre es actuar, y que la acción verbal que típicamente realizamos es compleja y se estructura en varios subactos entreverados:

RG Realizatividad generalizada: Hablar es siempre realizar actos (entrelazados).

Por otra parte, a lo largo Austin (1962) se adopta un principio holístico que, en apariencia, complementa al anterior, según el cual muchos problemas tradicionales de la filosofía del lenguaje se resuelven aplicando a los fenómenos una mirada abarcadora, alejada de las abstracciones usuales. Ese principio se formula de modo conciso en la última conferencia, como una moraleja de todas sus reflexiones anteriores (ibid., p. 148):

H Holismo austiniano: El acto de habla total en la situación de habla total es el único fenómeno real que estamos tratando de elucidar.

La línea de crítica que vamos a explorar se basa en atender a una tensión latente entre RG y H. Mientras que RG dicta que el habla debe explicarse como una estructura de actos entretejidos (lo que hace natural postular actos perlocucionarios en paralelo con los locucionarios y los ilocucionarios), $\mathrm{H}$ incorpora el importante concepto de situación de habla total como algo distinto del acto de habla total.22 Finalmente prevalece RG, lo que deja fuera de foco la importancia de la situación y su irreducibildad a las acciones del emisor. La noción de perlocución resalta (en exceso) los aspectos del habla que se dejan tratar como actos del hablante (si bien

bles taxonomías útiles adicionales a las exploradas. Por ejemplo, en la nota 6 se ha sugerido una clasificación de las perlocuciones en "positivas", "negativas" y "neutrales" para la audiencia.

22 No pretendo sugerir que esta diferencia sea perfectamente clara, que podamos distinguir nítidamente los aspectos que pertenecen esencialmente a un acto de otros aspectos que lo rodean. 
podrían ser tratados de otro modo), lo que tiene el efecto perverso de hacer poco visibles para la mirada teórica otros aspectos que tienen una importancia equivalente pero que no se dejan tratar como actos del emisor.

\subsection{El marco de una emisión}

Considérense los ejemplos incluidos en la siguiente tabla:

1)

\section{antecedentes}

$h$ se siente agraviado por $o \rightarrow$

$h$ siente animadversión hacia $o$

\section{ilocución}

$h$ insulta a $o \quad \rightarrow$

\section{consecuencias}

$o$ se siente ofendido por $h$ $o$ se siente herido por $h$

2)

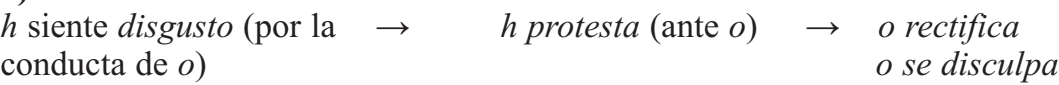

3) $h$ es generoso / $h$ está en deuda con $o / \quad h$ se siente obligado a hacer $p /$ $h$ aprecia a $o / h$ cree que $o$ desea $p \quad h$ intenta hacer $p / h$ hace $p$

$o$ desea de que $h$ haga $p /$

$\rightarrow \quad h$ promete que $p \rightarrow$ $o$ espera que $h$ haga (o intente hacer) $p$

/ o cree que $h$ está obligado a hacer $p$ / $o$ se alegra / $o$ queda agradecido

4) 23

$h$ desea vengarse de $o$

$h$ se siente satisfecho (ufano, jactancioso)

$h$ es un matón

$h$ siente remordimientos

$o$ teme a $h$

$\rightarrow \quad$ h amenaza a $o$ (con que $p$ ) $\rightarrow$

$o$ se asusta, se intimida, se siente humillado

$o$ siente ira, se resiste, se rebela.

5)

$h$ percibe dudas o ignorancia en $o /$

$h$ se siente satisfecho por impartir

$h$ desea (tal vez de modo interesado)

información útil (para $o, h$, o ambos)

que $o$ sepa que $p$

$\rightarrow$ h informa a $o$ de que $p \rightarrow$

$o$ desea saber algo, siente curiosidad

$o$ se convence / se persuade /

$o$ hace una pregunta.

se disuade de que $p$

23 Este ejemplo representa a una amenaza elemental (o no condicional) como “¡Te mataré!" (cfr. Blanco Salgueiro, 2010). Se recogen dos marcos alternativos, en lo que a los antecedentes que conciernen al hablante se refiere, el matonismo y el deseo de venganza. El matonismo es un rasgo del carácter, más que un tipo de estado mental, pero no entraremos en esas sutilidades, ya que se quiere dejar abierta la cuestión acerca de qué tipos de cosas puede incluir un marco situacional. 
En cada uno de los ejemplos, una ilocución aparece flanqueada por una serie de posibles antecedentes y consecuencias. Se destacan las emociones y otros estados mentales del hablante y del oyente y se ignoran, por simplicidad, otros posibles factores relevantes relacionados con terceras personas o con el mundo social y físico que, sin duda, deberían incluirse en una articulación más completa de un marco. Nos referiremos a los antecedentes, consecuencias y otras circunstancias de una emisión como su marco situacional y consideraremos como una parte importante del mismo el marco cognitivo-emocional formado por los estados mentales del hablante y del oyente anteriores y posteriores a la producción de dicha emisión. Algunos de los antecedentes señalados se incluyen en la TAH ortodoxa (searleana) como condiciones preparatorias o condiciones de sinceridad, mientras que muchas consecuencias están entre los típicos "efectos perlocucionarios" constitutivos de lo que ortodoxamente se trata como perlocuciones.

Inspirándonos en la distinción de Ted Cohen entre perlocuciones asociadas y no asociadas, podemos distinguir ahora entre el marco situacional asociado, formado por los antecedentes y consecuencias de una emisión cuya mención parece precisa para caracterizar de modo exhaustivo la propia ilocución (que será desafortunada a menos que venga precedida de tales antecedentes y tenga como objetivo producir tales consecuencias), y el marco situacional no asociado, que contendrá antecedentes y consecuencias que son sólo contingentes o que no están constitutivamente ligados a la ilocución aunque pueden ser parte importante de la situación de habla en casos particulares. La distinción entre componentes asociados y no asociados de un marco puede no ser nítida, ya que algunos componentes no estrictamente asociados a la ilocución pueden formar parte de situaciones de habla prototípicas. En principio, puede admitirse toda la borrosidad, textura abierta, o relativización a intereses explicativos que se considere necesaria (si bien a riesgo de aminorar la solidez de la noción teórica de marco situacional).

Los dos primeros ejemplos recogen sólo elementos del marco situacional asociado, respectivamente, a los actos ilocucionarios de insultar y de protestar. Puede contrastarse el primero de esos casos con aquel en el que $h$ insulta a $o$ porque se aburre (antecedente no asociado) y en el que $o$ (además de -o en lugar de- resultar ofendido) resulta sorprendido por $h$ (consecuencia no asociada), algo que seguramente ocurre en muchos casos, ya que uno pocas veces se espera un insulto. El resto de ejemplos incluye también elementos de posibles marcos no asociados pero típicos: uno fácilmente se imagina ilocuciones enmarcadas de esos modos. Por supuesto, no se pretende, a través de esos ejemplos esquemáticos, ofrecer un análisis completo de los marcos situacionales que rodean o pueden rodear a los actos de habla en cuestión. En principio, los marcos asociados deberían poder ser caracterizados de un modo exhaustivo, al menos en la medida en que los correspondientes "juegos de lenguaje" tengan perfiles bien delimitados (lo que, claro está, no admitiría un 
segundo-wittgensteiniano), por lo que indudablemente caen bajo el dominio de estudio de la TAH. Los componentes no asociados potenciales son infinitos e impredecibles, por lo que podemos preguntarnos si la TAH tiene que prestarles una atención especial. ${ }^{24}$

3.3. Tres argumentos contra las perlocuciones y a favor de los marcos situacionales

A continuación, se formulan tres argumentos a favor de sustitución de la noción de perlocución por la de marco situacional. La idea común a los tres es que la atención a las llamadas "perlocuciones" oculta una serie de importantes simetrías dentro del AHT-SHT, haciendo aparecer como muy heterogéneos fenómenos que en realidad no lo son.

\subsubsection{La simetría entre los antecedentes y las consecuencias}

En primer lugar, al otorgar una importancia especial a ciertas consecuencias de las emisiones, dándoles el nombre especial de "efectos perlocucionarios", se crea una distinción conceptual que distorsiona la simetría real entre las consecuencias y los antecedentes de una emisión. En general, las consecuencias de una ilocución se dejan tratar en el lenguaje común como actos del hablante: $h$ convenció a $o, h$ ofendió a $o$, aunque ello no es necesario y a veces resulta muy forzado y artificial hacerlo: $h$ hizo que o obedeciera, h provocó (con su pregunta) la respuesta $r$ de $o$. En cuanto a los antecedentes, no se dejan tratar en absoluto como acciones del emisor y, por ese motivo, muchos de ellos desaparecen simplemente para la mirada teórica guiada (o sesgada) por RG. Otros antecedentes (los asociados) son demasiado importantes para ser ignorados, por lo que la TAH ortodoxa los incluye como condiciones preparatorias o condiciones de sinceridad de una ilocución. En cualquier caso, se crea una brecha conceptual donde la realidad del habla parece ofrecer una situación muy simétrica: antes de la emisión, tenemos estados mentales del hablante y del oyente (además de otros factores) que la motivan o la contextualizan; después de la emisión, tenemos estados mentales del hablante y del oyente (y otros factores) que se siguen de ella y/o se persiguen a través de ella. La TAH no debería situar en lugares tan dispares de la teoría (psicología de $h$ y de $o v s$. condiciones de éxito $v s$. perlocuciones) elementos de naturaleza tan similar. La insistencia en la

\footnotetext{
${ }^{24} \mathrm{La}$ idea austiniana de que en circunstancias especiales una ilocución puede dar lugar a prácticamente cualquier tipo de perlocución (Austin, 1962, p. 110) puede reconvertirse ahora en la idea de que en circunstancias especiales podemos incluir prácticamente cualquier cosa entre los antecedentes y consecuentes no asociados a una ilocución.
} 
noción de perlocución conlleva un doble riesgo: o bien lleva a ignorar los antecedentes de una emisión, dejándolos fuera del dominio de la teoría del uso del lenguaje, o bien los remite a la teoría de las condiciones de éxito, de manera que son tratados de un modo muy diferente a como se tratan las consecuencias. Pero ¿por qué una teoría del uso del lenguaje va a estar menos interesada (o interesada de un modo diferente), por ejemplo, en las emociones que inducen a $h$ a insultar a $o$ que en las que resultan en $o$ como efecto del insulto (véase el primer ejemplo de la tabla)? La decisión parece arbitraria: o ambas deben quedar fuera de la teoría, limitando así el alcance de $\mathrm{H}$, o hay que incluirlas a las dos. Pero si se incluyen ambas, debería hacerse de un modo homogéneo, ya que lo que parece involucrado en ambos casos es que el habla se halla atravesada por emociones y otros estados mentales (y por circunstancias de todo tipo). ${ }^{25}$ La noción de marco quiere restituir la simetría intuitiva entre los estados mentales (en general, entre las circunstancias) antecedentes y subsiguientes a un acto de habla.

En cuanto a si tanto los marcos asociados como los no asociados deben formar parte del dominio del estudio pragmático del lenguaje, ello depende de cómo se conciba tal estudio. Podría ser pertinente prestar atención a ambos si la pragmática se concibe de un modo suficientemente amplio ( $c f r$. Verschueren, 1999, Introducción), aunque tienen un interés especial los marcos que resultan imprescindibles para explicar las ilocuciones, los asociados. Así, el primer ejemplo recoge la idea de que los insultos son ilocuciones motivadas por una emoción negativa dirigida al oyente y que, a la vez, intentan inducir un estado mental negativo en éste. No es posible entender el uso "insultivo" del lenguaje sin referirse a un marco así. En ese sentido, el estudio de los marcos asociados forma parte de una teoría de la fuerza, un motivo adicional para prescindir de la noción de perlocución. Los ejemplos 3-5 incluyen antecedentes y consecuencias que parecen contingentes y que, por tanto, tal vez no deberían considerarse como parte del núcleo explicativo de la TAH, aunque pueden tener interés para un estudio más holístico del lenguaje que tenga como dominio el estudio del habla en toda su complejidad. En la medida en que algunos de esos componentes sean típicos o estén ligados a ciertas situaciones interesantes, su estudio equivaldrá a algo más que a una casuística.

\footnotetext{
25 Cavell (2005) reivindica el papel de las emociones en el habla, considerando que Austin no les concedió la suficiente importancia. Su estrategia consiste en formular condiciones de felicidad para las perlocuciones análogas a las que Austin propone para el realizativo. Sin embargo, la estrategia de Cavell parece equivocada, ya que lo más importante del "habla apasionada" son los antecedentes emocionales, que no se dejan tratar fácilmente (o en absoluto) como aspectos del acto total del emisor, aunque sin duda pertenecen a la situación total de habla. La noción de "marco emocional" puede responder de un modo más adecuado al interés teórico por el estudio del aspecto emocional del habla.
} 


\subsubsection{La simetría entre las consecuencias de las ilocuciones exitosas y fallidas}

Un segundo argumento contra la noción de perlocución se basa en una nueva asimetría conceptual generada por ella que, de nuevo, parece infundada. Algunos estudios empíricos "multidimensionales" de actos de habla especiales como las promesas y las amenazas condicionales consideran que una dimensión importante en dichos actos es la emotiva, pero prescinden completamente de la noción de perlocución (ya que no se enmarcan dentro de la TAH) y se concentran sin más en las emociones que parecen estar en juego ( $c f r$. Beller, 2002; Beller \& Bender, 2004; Beller, Bender \& Kuhnmünch, 2005). Este tipo de enfoques refleja el rumbo natural que puede seguir un estudio holístico del habla liberado de prejuicios terminológicos, ya que no sólo tienen en cuenta las emociones ("asociadas") ligadas a la realización con éxito de las correspondientes ilocuciones, alegría, en el caso de las promesas, miedo o ira, en el de las amenazas, sino que atienden también a las emociones desencadenadas cuando los actos de habla no tienen éxito o se desactivan, alivio en el caso de las amenazas, tristeza, frustración, etc. en el de las promesas. Puesto que lo perlocucionario tiene que ver sólo con las consecuencias de las ilocuciones exitosas, el concepto no cubre teóricamente este último aspecto de las posibles situaciones totales de habla. Pero, de nuevo, esto no parece justificado, si de lo que se trata es de ofrecer una visión contextualizada del habla. ¿Por qué la intimidación que produce una amenaza debe considerarse más importante teóricamente que el alivio que produce su desactivación? Una noción adecuada de marco (más articulada que la esbozada en nuestra tabla de ejemplos) debería tener en cuenta ambas cosas como parte de la explicación del juego de lenguaje de amenazar y de las formas de vida en cuyo seno cobra sentido. Parece tan asociado a una amenaza (tan constitutivo de ella) el propósito de producir intimidación en la audiencia como que su desactivación produce una sensación de alivio en dicha audiencia. Y si queremos incluir en nuestro estudio las consecuencias no asociadas de una ilocución, parece que deberíamos incluir también las consecuencias no asociadas de su desactivación (por ejemplo, la desactivación de una amenaza puede producir sorpresa en la audiencia). En resumen, un segundo argumento contra las perlocuciones y a favor de los marcos apela a la paralela relevancia teórica de los efectos de las ilocuciones fallidas en relación a los efectos de las ilocuciones exitosas, paralelo que la noción de perlocución contribuye a ocultar.

\subsubsection{La simetría entre la actividad del hablante y la de la audiencia}

El tercer argumento contra las perlocuciones concierne a una nueva asimetría problemática que esa noción teórica genera entre los papeles del hablante y del oyente. Tratar como parte de los actos de $h$ los sucesos ejemplificados en la parte 
derecha de nuestra tabla produce una visión de la situación típica de habla en la que la audiencia se introduce sólo de contrabando y aparece como pasiva, cuando lo que ocurre en muchos casos paradigmáticos es que ella es la principal responsable del evento en cuestión, la que crea, a menudo muy activamente, el supuesto "efecto perlocucionario". Considerar la contribución de la audiencia como algo importante de suyo requiere tratarla como parte activa de la situación total de habla, y no como algo supeditado a la acción del hablante. ${ }^{26}$ Esto es así tanto si el efecto es intencionado por parte de $h$ como si no lo es, pero se manifiesta más claramente en el segundo caso. Imaginemos que $h$ promete que se casará con $o$ intentando que $o$ se alegre pero que sólo consigue deprimirlo/a. Podemos hablar de que $h$ deprimió a $o$, y esa forma de hablar puede ser útil en algunos contextos de la vida ordinaria. Pero desde un punto de vista teórico, para explicar lo que ha ocurrido y por qué se ha producido ese resultado, debemos considerar la situación de un modo global y proceder a un examen de las creencias, sentimientos y emociones conscientes o inconscientes, y procesos de razonamiento de $o$. Las palabras de $h$ sólo tienen un valor secundario en la explicación, como causa desencadenante de un proceso que tiene su parte principal en la mente de la audiencia.

La conclusión, aparentemente similar a la nuestra, a la que llega Gu (1993), que consiste en negar lo que denomina "tesis de la causación", según la cual los hablantes causan efectos perlocucionarios en la audiencia, se basa en una idea demasiado estrecha de causa, como causa desencadenante (triggering cause). Una noción menos restrictiva permite reivindicar el papel activo de la audiencia a la vez que nuestra noción de marco. La idea de Gu es que la llamada "causa perlocucionaria" sólo puede ser la causa desencadenante de los llamados "efectos perlocucionarios", pero que lo que resulta más relevante en el caso típico son los procesos de razonamiento en la audiencia que, por ejemplo, pueden llevarla, (ante la afirmación, por parte de $h$, de que $p$ ) a convencerse por su cuenta de que $q$. Según $\mathrm{Gu}$, las causas no son condiciones necesarias ni suficientes para el efecto perlocucionario, sino que tienen "sólo" el papel de causas desencadenantes en condiciones normales. Así, decirte "Hay una araña en tu solapa" sólo conseguirá el efecto de asustarte si tienes miedo a las arañas (y crees que digo la verdad, etc.), pero si coleccionas arañas, tal vez lo que ocurra es que te alegres. La noción de marco, y en particular la de ante-

\footnotetext{
26 Este sesgo monologista de la teoría de Austin (que, para ser justos, a menudo concede a la audiencia un papel importante, al menos en lo concerniente a las ilocuciones), se radicaliza en la TAH searleana y de inspiración griceana. La crítica a la suposición de la pasividad de la audiencia centra la argumentación de Gu (1993), y se formula también en Campbell (1973):

[Austin] might have gone on to say that in the "total speech situation" both speaker and listener act, rather than implying that the speaker acts while the listener is acted on, that there is an activepassive dichotomy in which to urge, persuade, compliment, or what you are acts, while to be urged, persuaded, complimented are not acts at all, but mere passive responses (Campbell, 1973, p. 292).
} 
cedente, puede de nuevo ayudarnos aquí, combinada con la distinción de Fred Dretske entre las causas desencadenantes y las causas estructurantes de un suceso. Según Dretske (1987), cuando preguntamos por qué sucedió algo, por ejemplo, por qué ardió el pajar, podemos responder con la causa que puso en marcha el proceso ("alguien arrojó una colilla mal apagada") o con las condiciones que lo hicieron posible ("la paja estaba muy seca"). Ambas respuestas son correctas y podemos estar interesados en una u otra, según el caso. Nuestro tercer argumento contra la noción de perlocución puede reformularse entonces diciendo que esa noción conduce a una concentración excesiva en la causa desencadenante, situada en la mente o en las palabras del hablante, y a dejar de lado la causa estructurante, constituida en gran medida por factores activos y pasivos, antecedentes y subsiguientes a la ilocución, dependientes de la audiencia (y de otros factores ajenos al control del emisor). Hechos como que $o$ tenga miedo a las arañas o, alternativamente, que coleccione arañas, pueden tratarse como causas estructurantes (como parte de los antecedentes del marco), por contraste con la causa desencadenante (la ilocución). Considerar en serio a la audiencia como parte de la situación total de habla y darle un papel activo en la explicación de por qué alguien se convenció, se alegró, etc. requiere abandonar la consideración del habla como un acto unilateral del hablante y a adoptar un enfoque más interactivo.

\section{Conclusiones}

Se ha argumentado que la noción de perlocución es problemática si lo que se busca es forjar un concepto teórico que permita dar pasos firmes hacia la elucidación del acto de habla total en la situación de habla total (y, en particular, de los aspectos cognitivo-emocionales involucrados), según aconseja el principio holista austiniano. Al poner el foco en ciertos supuestos actos del emisor, la noción no refleja adecuadamente tres importantes simetrías en la situación total: la simetría entre los antecedentes y las consecuencias de una ilocución; la simetría entre las respuestas de la audiencia ante el éxito o cumplimiento y ante el fracaso o desactivación de una ilocución; y la simetría entre los papeles activos en el discurso del hablante y del oyente. Se mostró también que el intento de reconstruir la noción de perlocución distinguiendo múltiples tipos que permitan resolver las ambigüedades incrustadas en ella acaba creando un constructo de formidable complejidad. La noción de "marco situacional de una emisión" remite a una estructura capaz de capturar de modo natural las tres simetrías mencionadas, por lo que podría ser el concepto que la TAH necesita coordinar con el de ilocución para sus fines explicativos fundamentales. 27

\footnotetext{
27 Aunque una de las principales ventajas de la noción de marco es la de destacar ciertas simetrías en el AHT-SHT, en principio permite representar también las asimetrías que se puedan detectar.
} 
La propuesta final de este trabajo es, por tanto, prescindir de la noción de perlocución como concepto teórico fundamental para la TAH y cubrir los fenómenos a los que parece apuntar esa noción con el concepto más general de marco situacional. Supone pues, una solución "eliminativa" (prescindamos de la noción), o tal vez sería más exacto llamarla "sustitutiva" (reconceptualicemos los fenómenos a los que apunta la noción), al problema de las perlocuciones. Ello requeriría resituar también la teoría de las condiciones de felicidad, como parte de la caracterización de los antecedentes (asociados) de un marco. La propuesta es, pues, bastante revisionista.

No se ha querido sugerir que Austin previese para las perlocuciones un papel que, en realidad, no pueden cumplir. Probablemente las introdujo como un concepto tentativo y de valor explicativo limitado. Un modo de reconciliar hasta cierto punto las dos vías exploradas podría pasar por retener la noción de perlocución (o alguna de sus modalidades) a la vez que se introduce la noción más global de marco, utilizando la primera de un modo modesto (y no como un concepto teórico central) cuando estemos especialmente interesados en la "causa desencadenante". Ello sucede cuando queremos atribuir al hablante responsabilidad por un suceso ulterior a su ilocución, porque intentó producirlo o porque debería haberlo previsto. En el ámbito penal se tiene en cuenta no sólo si alguien amenazó a $o$, sino si lo intimidó o si le produjo "secuelas psicológicas". Pero también en la vida cotidiana muchas veces estamos más interesados en las causas que desencadenan ciertos procesos que en las causas estructurantes que los hacen posibles, lo que explica la existencia en las lenguas naturales de multitud de verbos "perlocucionarios" como convencer, ofender, intimidar, etc. Sin embargo, no debemos despreciar la importancia ordinaria de las causas estructurantes, que constantemente invocamos al decir cosas como: "No me intimidas, pues no tengo nada que temer de ti", "No soy el tipo de persona que se deja convencer por argumentos demagógicos", etc.

Queda en el aire la cuestión de precisar la noción de marco situacional de modo que se pueda hacer un uso teórico de ella en la explicación de una gama de aspectos del habla. De acuerdo con lo dicho, tal elaboración debe cumplir al menos los siguientes requisitos: 1) Debe permitir distinguir los componentes asociados de los no asociados del marco (aceptando que los límites entre ambos pueden ser borrosos). 2) Debe permitir distinguir los componentes situacionales de un marco que tienen que ver con el éxito de las ilocuciones de los que conciernen a su posible fracaso. 3) Debe incluir tanto los componentes de un marco que dependen sólo del hablante como los que dependen principalmente de la actividad de la audiencia (o, en general, de factores situacionales que no sobrevienen a la mente y a los actos del emisor). 


\section{Referencias bibliográficas}

Austin, J. L. (1956/57): “A plea for excuses”, Proceedings of the Aristotelian Society, 57, pp. 1-30.

Austin, J. L. (1962): How to do Things with Words, Nueva York, Oxford University Press (2nd ed., 1975). [Traducción al español de Genaro R. Carrió y Eduardo A. Rabossi: Cómo hacer cosas con palabras, Barcelona, Paidós, $1^{a}$ ed., 1971.]

BaCH, K. y Harnish, R. M. (1979): Linguistic Communication and Speech Acts, Cambridge, Mass., MIT Press.

Beller, S. 2002. "Conditional promises and threats - Cognition and emotion", Proceedings of the Twenty-Fourth Annual Conference of the Cognitive Science Society, Mahwah, NJ: Lawrence Erlbaum, pp. 113-118.

Beller, S. and Bender, A. (2004): "Cultural differences in the cognition and emotion of conditional promises and threats - Comparing German and Tonga", en K. D. Forbus, D. Gentner y T. Regier (eds): Proceedings of the Twenty-Sixth Annual Conference of the Cognitive Science Society, Mahwah, NJ: Lawrence Erlbaum, pp. 85-90.

Beller, S., Bender, A. and Kuhnmünch, G. (2005): "Understanding conditional promises and threats", Thinking \& Reasoning, 11/3, pp. 209-238.

BERLIN, I. et al. (1973): Essays on J. L. Austin, Oxford, Clarendon Press.

Blanco Salgueiro, A. (2004): Palabras al viento. Ensayo sobre la fuerza ilocucionaria, Madrid, Trotta.

Blanco SAlgueiro, A. (2008): "Cómo hacer cosas malas con palabras: actos ilocucionarios hostiles y los fundamentos de la teoría de los actos de habla", Crítica 40/118, pp. 3-27.

Blanco Salgueiro, A. (2010): "Promises, threats, and the foundations of Speech Act Theory", Pragmatics, 20/2, pp. 213-228.

CAmpBell, P. N. (1973): "A rethorical view of locutionary, illocutionary and perlocucionary acts", Quarterly Journal of Speech, 59, pp. 284-296.

CAVELL, S. (2005): "Performative and passionate utterance", en S. Cavell: Philosophy the Day After Tomorrow, Cambridge/Londres: Harvard University Press, pp. 155-191.

CERF, W. (1969): "Critical review of How to do Things with Words", en Fann (ed.), pp. 351-379.

CoHEn, L. J. (1969): “Do illocutionary forces exist?”, en Fann (ed) (1969), pp. 420444.

CoHEN, T. (1973): "Illocutions and perlocutions", Foundations of Language, 9, 492503.

DAvis, S. (1979): "Perlocutions", en J. R. Searle, F. Kiefer \& M. Bierwish (eds.): Speech Act Theory and Pragmatics, Amsterdam, Reidel, pp. 37-55. 
DRETSKe (1987): Explaining Behaviour. Reasons in a World of Causes, Cambridge, Mass, MIT Press.

Fann, K. T. (ed.) (1969): Symposium on Austin, Londres, Routledge \& Kegan Paul.

GAINES, R. N. (1979): "Doing by saying: toward a theory of perlocution". Quarterly Journal of Speech, 65, pp. 207-217.

Gu, Y. (1993): "The impasse of perlocution", Journal of Pragmatics, 20, pp. 405432.

Hadley Chase, J. (1960): Una corona para tu entierro, Madrid, Punto de lectura, 2008.

Hornsby, J. (1993): "Speech acts and pornography", Women's Philosophical Review, Noviembre, pp. 38-45.

Hornsby, J y Langton, R. (1998): "Free Speech and Illocution". Legal Theory 4, pp. 21-37.

KuRZON, D. (1998): “The speech act status of incitement: perlocutionary acts revisited", Journal of Pragmatics, 29, pp. 571-596.

Langton, R. (1993): "Speech acts and unspeakable acts", Philosophy and Public Affairs, 22, pp. 293-330.

Lewis, D. (1969): Convention. A Philosophical Study. Cambridge, Mass, Harvard University Press.

Nicoloff, F. (1989): “Threats and illocutions", Journal of Pragmatics, 13, pp. 501522.

Searle, J. R. (1969): Speech Acts. An Essay in the Philosophy of Language, Cambridge, Cambridge University Press. [Traducción al español de L.M. Valdés Villanueva: Actos de habla. Ensayo de filosofia del lenguaje, Madrid, Cátedra, 1994.]

SEARLE, J. R. (1973): “Austin on locutionary and illocutionary acts”, en Berlin et al. (1973), pp. 141-159.

Searle, J. R. (1983): Intentionality. An Essay in the Philosophy of Mind, Cambridge, The Syndicate of the Press of the University of Cambridge. [Traducción al español de E. Ujaldón Benítez, revisada por L. M. Valdés Villanueva: Intencionalidad. Un ensayo en la filosofia de la mente, Madrid, Tecnos, 1992.]

Searle, J. R. (1998): Mind, Language and Society. Philosophy in the Real World, Nueva York, Basic Books [Traducción al español de Jesús Alborés: Mente, lenguaje y sociedad. La filosofía en el mundo real. Madrid: Alianza, 2001.]

Strawson, P. F. (1964): "Intention and Convention in Speech Acts", reimp. en P. F. Strawson (1971): Logico-Lincuistic Papers, London, Methuen, pp. 149-169.

StraWson, P. F. (1973): “Austin and 'locutionary meaning", en Berlin et al. (1973), pp. 46-68.

Verschueren, J. (1999): Understanding Pragmatics, Londres, Edward Arnold Publishers. 
Wittgenstein, L. (1997): Philosophische Untersuchungen / Philosophical Investigations, Oxford, Blackwell.

Antonio Blanco Salgueiro

Dpto. de Lógica y Filosofía de la Ciencia

Facultad de Filosofía

Universidad Complutense de Madrid

ablancos@ucm.es 